\title{
Influence of Dodecyltrimethylammonium Halides on Thermotropic Phase Behaviour of Phosphatidylcholine/Cholesterol Bilayers
}

\author{
Bożenna Różycka-Roszak* and Hanna Pruchnik \\ Agricultural University, Department of Physics and Biophysics, Norwida 25, \\ 50-375 Wrocław, Poland. Fax: +4871 3205172. E-mail: Boro@ozi.ar.wroc.pl \\ * Author for correspondence and reprint requests \\ Z. Naturforsch. 55c, $753-757$ (2000); received March 27/May 11, 2000 \\ Counterions, Phosphatidylcholine Bilayers, Cholesterol \\ Effects of dodecyltrimethylammonium chloride (DTAC), dodecyltrimethylammonium bro- \\ mide (DTAB) and dodecyltrimethylammonium iodide (DTAI) on thermotropic phase behav- \\ iour of phosphatidylcholine bilayers containing cholesterol as well as on ${ }^{1} \mathrm{H}$ NMR spectra \\ were studied. Two series of experiments were performed. In the first one the surfactants \\ were added to the water phase while in the other directly to the lipid phase (a mixed film \\ from cholesterol, surfactant and phosphatidylcholine was formed). The effects of particular \\ surfactants on the main phase transition temperature, $T_{\mathrm{m}}$, were more pronounced when \\ added to the lipid phase ( $2^{\text {nd }}$ method) than to the water phase ( ${ }^{\text {st }}$ method); the opposite \\ happened when cholesterol was absent (Różycka-Roszak and Pruchnik 2000, Z. Naturforsch. \\ 55 c, 240-244). Furthermore, in the case of the first method the transitions were asymmetrical \\ while in the second method nearly symmetrical. It is suggested that surfactant poor and \\ surfactant rich domains are formed when surfactants are added to the water phase.
}

\section{Introduction}

In the preceding paper (Różycka-Roszak and Pruchnik, 2000) we studied the influence of chloride, bromide and also iodide as counterions on the effect of amphiphilic compounds on thermotropic phase behaviour of phosphatidylcholine bilayers. Accordingly, we studied the commonly used surfactants like dodecyltrimethylammonium chloride (DTAC), dodecyltrimethylammonium bromide (DTAB) and dodecyltrimethylammonium iodide (DTAI). The objective of the present paper was to study the influence of the same dodecyltrimethylammonium halides (DTAX) on thermotropic phase behaviour of phosphatidylcholine bilayers containing cholesterol (DPPC/chol). Such studies seem to be interesting because cholesterol is a major component of the plasma membranes in many cells of higher organisms. A number of papers are devoted to the effects of cholesterol incorporation on the properties of phospholipid bilayers, also on thermotropic phase behaviour (Cullis et al., 1978; McIntosh, 1978; Mouritsen, 1991).

As before, two series of experiments were performed. In the first one the surfactants were added to the water phase and in the other directly to the lipid phase (mixed film was formed).
Also as before, we applied differential scanning calorimetry (DSC), which is widely used to study the effects of cholesterol (McMullen et al., 1993; McMullen and McElhaney, 1995; McMullen et al., 1999), anesthetics, drugs, proteins (Papahadjopoulos et al., 1975) surfactants (Grau et al., 1999) and various small molecules on phase transitions of phospholipids. Besides, as before we used the ${ }^{1} \mathrm{H}$ NMR method; which, like other nuclear magnetic resonance methods, is one of the most powerful techniques (Fenske, 1993; Wu, 1996; Watss and Spooner, 1991) that have been applied to study biological and model membranes.

\section{Materials and Methods}

\section{Chemicals}

1,2-Dipalmitoyl-sn-Glycero-3-Phosphocholine (DPPC), cholesterol and egg yolk lecithin were purchased from Avanti Polar Lipids, Birmingham, Alabama.

Dodecyltrimethylammonium chloride (DTAC) and dodecyltrimethylammonium bromide (DTAB) were purchased from Fluka Chemie AG, Switzerland.

Dodecyltrimethylammonium iodide (DTAI) was prepared as before (Różycka-Roszak and Pruchnik, 2000). 
99.98\% $\mathrm{D}_{2} \mathrm{O}$ was purchased from Dr. Glaser AG Basel.

\section{Sample preparation for differential scanning} calorimetry (DSC)

Samples for DSC were performed on multilamellar vesicles (MLVs) which were prepared using two methods as described before (Różycka-Roszak and Pruchnik, 2000) with the difference that to DPPC $5 \mathrm{~mol} \%$ of cholesterol was added. The same DSC calorimeter was used as before.

\section{Sample preparation for ${ }^{1} H N M R$}

Measurements were performed on sonicated vesicles (SUVs) which were prepared using two methods as described before (Różycka-Roszak and Pruchnik, 2000) with the difference that to egg lecithin $5 \mathrm{~mol} \%$ of cholesterol was added. The rest of the procedure and spectrometer were as before.

\section{Results}

DSC

Some representative DSC plots of DPPC/chol. liposomes containing increasing amounts of DTAX prepared by means of the first and second method are compared in Figs. 1, 2 and 3, respectively. In the absence of a surfactant DPPC/chol. bilayers did not exhibit the pretransition since cholesterol is known to be an inhibitor of pretransition. The pretransition is known to be abolished by addition of very small amounts of cholesterol (McMullen and McElhaney, 1995).

The main phase transition temperature $\left(\mathrm{T}_{\mathrm{m}}\right)$ of DPPC/chol. liposomes prepared by means of the first and second method shifts progressively to lower temperatures and the transition broadens with increasing concentration of surfactants. Like it was for DPPC liposomes, in the case of the first method the transition peaks are broader and asymmetrical, while in the second method they are less broad and almost symmetrical. After addition of DTAX to DPPC/chol. liposomes according to the first method the main phase transition is not only asymmetrical but even often separates into two peaks. The high temperature peak can be ascribed to pure DPPC.

The effects of particular surfactants on $T_{m}$ were more pronounced when they were added to the

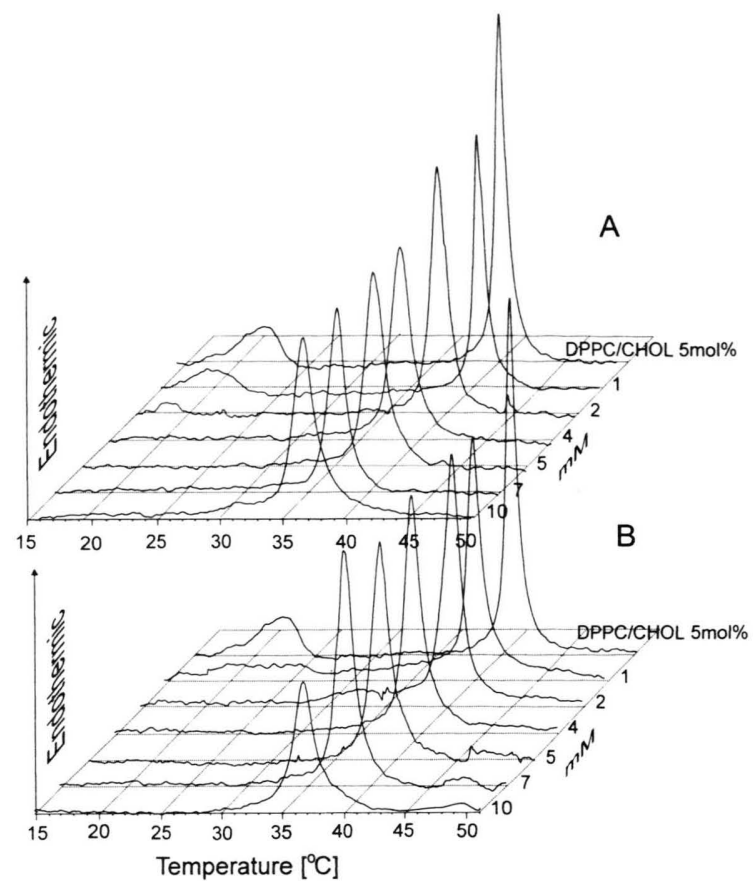

Fig. 1. DSC heating curves of MLVs with increasing molar ratio of dodecyltrimethylammonium chlorides (DTAC) to phosphatidylcholine/cholesterol (DPPC/ chol.). The curves were normalized for the amount of DPPC; (A) first method, (B) second method.

DPPC/chol. phase than to the water phase, while in the case of DPPC liposomes the effect was opposite. DTAC and DTAI show a stronger effect on $T_{m}$ in the case of the $2^{\text {nd }}$ method while DTAB approximately the same in both cases.

\section{${ }^{1} H N M R$}

The effects of DTAB, DTAC and DTAI on ${ }^{1} \mathrm{H}$ NMR spectra of egg lecithin/chol. liposome dispersion prepared according to the first and second method are compared in Fig. $4 .{ }^{1} \mathrm{H}$ NMR resonance of the trimethylammonium group of lecithin, $\left[\mathrm{N}\left(\mathrm{CH}_{3}\right)_{3}\right]_{\mathrm{L}}$, remains almost unchanged after addition of a surfactant. ${ }^{1} \mathrm{H}$ NMR resonance of the trimethylammonium group of surfactant head group head, $\mathrm{N}\left(\mathrm{CH}_{3}\right)_{3}$, is downfield shifted in liposomes dispersion and of significantly lower intensity in comparison to pure water. When surfactants are added to the lipid phase the intensity of ${ }^{1} \mathrm{H}$ NMR resonance of the trimethylammonium group of surfactant head group decreases more than when they are added to water phase (first 


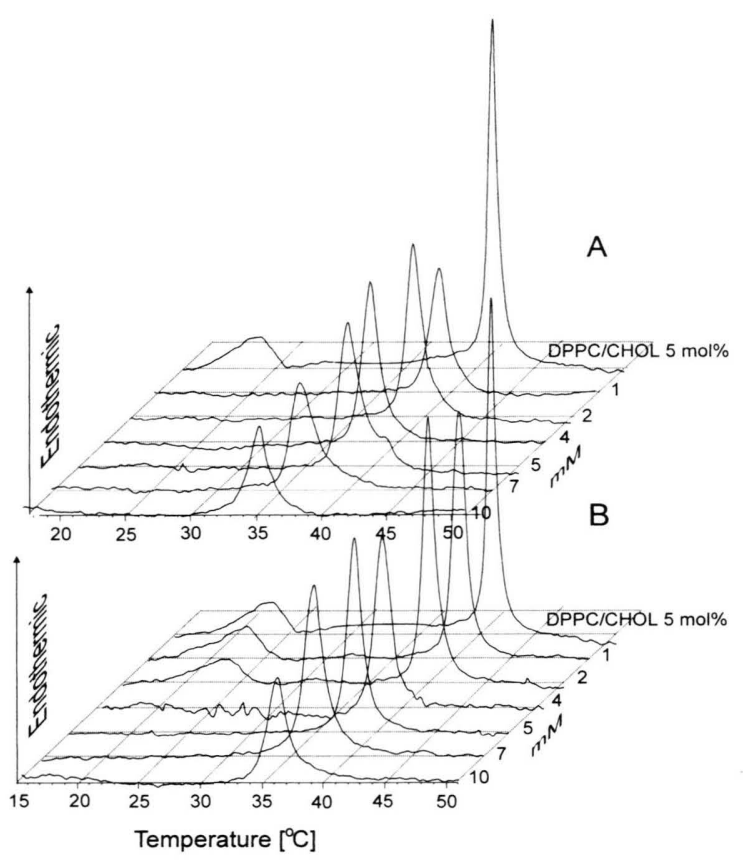

Fig. 2. DSC heating curves of MLVs with increasing molar ratio of dodecyltrimethylammonium bromide (DTAB) to phosphatidylcholine/cholesterol (DPPC/ chol). The curves were normalized for the amount of DPPC; (A) first method, (B) second method.

method). Besides, in the case of both the methods the intensity of $\mathrm{N}\left(\mathrm{CH}_{3}\right)_{3}$ signals coming from DTAC and DTAB are approximately the same while that coming from DTAI is significantly lower. This suggest that, like it was in the case of egg lecithin liposomes, that more surfactants are embedded into the lipid phase of egg lecithin/chol. liposomes upon addition according to the second method. Besides, in the case of both methods the strongest effect is for DTAI and for DTAC and DTAB approximately the same. After addition of DTAI to lipid phase the $\mathrm{N}\left(\mathrm{CH}_{3}\right)_{3}$ signals coming from the surfactant headgroup was so much downfield shifted that superimposed on the choline, $\left[\mathrm{N}\left(\mathrm{CH}_{3}\right)_{3}\right]_{\mathrm{L}}$ signal.

\section{Discussion}

The effects of the counterions studied on influence of dodecyltrimethylammonium halides on the phase transitions of DPPC/chol. liposomes as well as on ${ }^{1} \mathrm{H}$ NMR spectra of egg lecithin/chol. liposomes show some similarities with their effect on

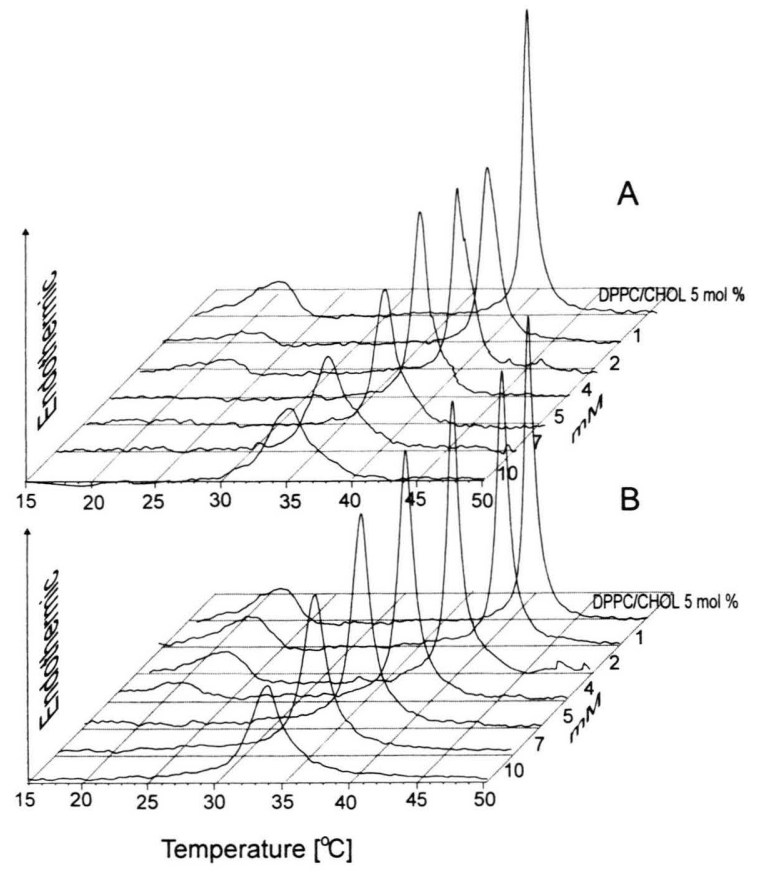

Fig. 3. DSC heating curves of MLVs with increasing molar ratio of dodecyltrimethylammonium iodide (DTAI) to phosphatidylcholine/cholesterol (DPPC/chol.). The curves were normalized for the amount of DPPC; (A) first method, (B) second method.

liposomes without cholesterol. In both the cases their effect depends on the way a surfactant was introduced to liposomes. In the case of the $1^{\text {st }}$ method of preparation the transitions were asymmetrical, while in the $2^{\text {nd }}$ method nearly symmetrical. Asymmetrical transitions may suggest that, like it was in the case of DPPC liposomes, surfactant-poor and surfactant-rich domains are formed. Anyway, in the presence of cholesterol the influence of a counterion on the interaction of a surfactant with lipid bilayer was more enhanced when the surfactant was added to the lipid phase than to water phase; the opposite happened when cholesterol was absent. (Różycka-Roszak and Pruchnik, 2000). This may indicate that in the presence of cholesterol the water-ion interactions are less significant as they were in the absence of cholesterol. Probably, more important are cholesterol surfactant interactions in which counterions are involved. When a surfactant was added to DPPC/ chol. liposomes through the water phase the effect of counterion on the phase transitions slightly 


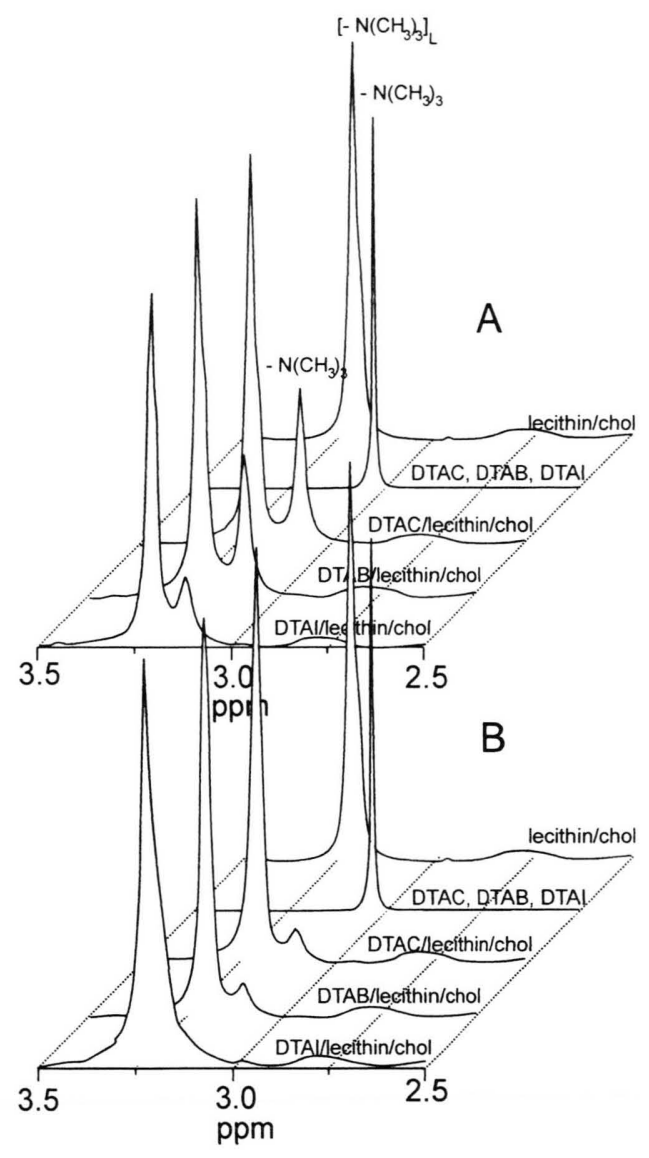

Fig. 4. ${ }^{1} \mathrm{H}$ NMR spectra of: egg yolk lecithin/cholesterol liposome suspension in the presence and absence of surfactants; $\left[\mathrm{N}\left(\mathrm{CH}_{3}\right)_{3}\right]_{\mathrm{L}}$ - trimethylammonium group of lecithin.

(A) first method (B) second method. Lecithin concentration $10 \mathrm{mg} \mathrm{cm}^{-3}$, surfactant concentration $2 \mathrm{~mm}$. increased in the order chloride $<$ bromide $<$ iodide. Also, when surfactants were added to DPPC liposomes according to the first method the same order was observed but the effect was much more pronounced. Anyway, the above sequence was changed when surfactants were added to DPPC/ chol. phase. Surprisingly, enhanced effect was observed for DTAC. This may indicate that there are stronger interactions between cholesterol and DTAC when the surfactant is added to liposomes according to the second method than according to the first one. This may be due to the fact that chloride in chloroform is not hydrated so that the smallest one of the halide studied can approach surfactant cation and enhance its interaction with cholesterol the most. In the water phase, chloride, being the most hydrated of the halide ions, can not approach the liposome surface so closely as the other counterions, and this is probably why the influence of DTAC on thermotropic phase behaviour of DPPC/chol. liposomes as well as on DPPC liposomes is the smallest.

\section{Acknowledgement}

This work was sponsored by the Polish Research Committee (KBN), grant no. PO4G 07712. 
Cullis P. R., Van Dijck P. W. M., De Kruijff B. and De Gier J. (1978), Effects of cholesterol on the properties of equimolar mixtures of synthetic phosphatidylethanolamine and phosphatidylcholine. $\mathrm{A}{ }^{31} \mathrm{P}$ NMR and differential scanning calorimetry study. Biochim. Biophys. Acta 513, $21-30$.

Fenske D. (1993), Structural and motional properties of vesicles as revealed by nuclear magnetic resonance. Chem. Phys. Lipids 64, 143-162

Grau A., Gómez Fernández J. C., Peypoux F. and Ortiz A. (1999), A study on the interactions of surfactin with phospholipid vesicles. Biochim. Biophys. Acta 1418, $307-319$.

McIntosh T. (1978), The effect of cholesterol on the structure of phosphatidylcholine bilayers. Biochim. Biophys. Acta 513, 43-58.

McMullen T. P. W., Lewis R. N. A.H. and McElhaney R. N. (1993), Differential scanning calorimetric study of the effect of cholesterol on the thermotropic phase behaviour a homologous series of linear saturated phosphatidylcholines. Biochemistry 32, 516-522.

McMullen T. P. W., Lewis R. N. A.H. and McElhaney R. N. (1995), New aspects of the interaction of cholesterol with dipalmitoylphosphatidylcholine bilayers as revealed by high-sensitivity differential scanning calorimetry. Biochim. Biophys. Acta 1234, 90-98.
McMullen T. P. W., Lewis R. N. A.H. and McElhaney R. N. (1999), Calorimetric and spectroscopic studies of the effects of cholesterol on the thermotropic phase behaviour and organization of a homologous series of linear saturated phosphatidylethanolamine bilayers. Biochim. Biophys. Acta 1416, 119-134.

Mouritsen O. G. (1991), Theoretical models of phospholipid phase transitions. Chem. Phys. Lipids 57, 179194.

Papahadjopoulos D., Moscarello M., Eylar F. H. and Isac T. (1975), Effects of proteins on thermotropic phase transitions of phospholipid membranes. Biochim. Biophys. Acta 401, 317-335

Różycka-Roszak B., Pruchnik H. (2000), Effect of counterions on the influence of dodecyltrimethylammonium halides on thermotropic phase behaviour of phosphatidylcholine bilayers. Z. Naturforsch. 55c, 240244.

Watts A. and Spooner P. J. R. (1991), Phospholipid phase transitions as revealed by NMR. Chem. Phys. Lipids 57, 195-211.

Wu W. (1996), Sonicated membrane vesicle. In: Encyclopedia of NMR. (D. M. Grant and R. K. Harris, ed.). John Wiley \& Sons, Ltd., Sussex, England, pp. 44854492. 